\title{
Saussure correcteur de Louis Duvau
}

\section{Marc Décimo}

\section{(2) OpenEdition}

\section{Journals}

\section{Édition électronique}

URL : http://journals.openedition.org/genesis/1091

DOI : 10.4000/genesis. 1091

ISSN : 2268-1590

\section{Éditeur :}

Presses universitaires de Paris Sorbonne (PUPS), Société internationale de génétique artistique littéraire et scientifique (SIGALES)

\section{Édition imprimée}

Date de publication : 15 novembre 2012

Pagination : 195-200

ISBN : 978-2-84050-869-4

ISSN : 1167-5101

\section{Référence électronique}

Marc Décimo, « Saussure correcteur de Louis Duvau », Genesis [En ligne], 35 | 2012, mis en ligne le 15 novembre 2014, consulté le 27 juin 2020. URL : http://journals.openedition.org/genesis/1091 ; DOI : https://doi.org/10.4000/genesis.1091 


\title{
Saussure correcteur de Louis Duvau
}

\author{
Marc Décimo
}

Le parcours de Louis Duvau, né à Saumur le 12 juillet 1864 et mort prématurément le 14 juillet 1903 à Angers, est un cas exemplaire de destin durant cette période d'institutionnalisation en France de la linguistique.

C'est probablement Anatole Bailly (1833-1911), le condisciple de Michel Bréal (1832-1915) à l'École normale supérieure, l'auteur du célèbre Dictionnaire grecfrançais et titulaire de la chaire de quatrième au lycée d'Orléans, qui repère Louis Duvau : ce dernier reçoit le prix de thème latin en quatrième ${ }^{1}$. C'est encore Bailly qui guide probablement Duvau d'Orléans vers Paris et vers Bréal2. Duvau concourt avec succès pour une des bourses de licence récemment fondées à l'Université de Paris et, dès 1882-1883, il fréquente l'École des hautes études en qualité de boursier de licence ${ }^{3}$. Licencié en 1883, agrégé de grammaire en 1884, il suit en 1884-1885 et 1885-1886 les cours de gotique de Ferdinand de Saussure.

Spécialisé en philologie latine, en grammaire comparée et en langues et littératures celtiques, Duvau est envoyé par l'École en 1885 à Wölfenbüttel et Leyde pour étudier des manuscrits latins de l'époque archaïque et, en 1886, à Vienne et à Prague; grâce à une subvention de la Ville de Paris, il passe ensuite un an à l'Université de Leipzig et on le retrouve membre de l'École française de Rome en 1887-1888. En 1888, Duvau est nommé maître de conférences à Dijon pour la philologie classique, puis en 1889-1890 à Lille; il donne trois conférences, dont une de gotique, et une bénévole de sanskrit - l'une de ces conférences n'est fréquentée que par un élève. Lorsque se libère le poste de Saussure parti définitivement s'installer à Genève en 1891, c'est Antoine Meillet qui est pressenti : n'avait-il pas remplacé Saussure en 1889 lorsque celui-ci avait pris un congé d'un an ?
Duvau fait cependant acte de candidature. Dans une lettre adressée à Gaston Paris, qui préside l'École, datée du 11 juin 1891 de Lille4, il écrit : «M. Havet m'a communiqué il y a quelque temps les réflexions fort justes que vous lui aviez soumises au sujet des conséquences pécuniaires qu'avait pour moi une nomination à l'École des Hautes-Études 5 . Il se trouve que grâce à certains arrangements, je suis en situation de me contenter, pendant quelques années au moins d'un traitement médiocre, et dans ces conditions, je n'hésite pas à poser ma candidature à la chaire devenue vacante par le départ de M. de Saussure. »

Duvau est bel et bien en lice. Dans une autre lettre à Gaston Paris, il fait part le 19 juin 1891 de son intention :

Je me vois forcé de venir vous importuner encore une fois. J'ai reçu dans l'après-midi une longue lettre de M. de Saussure,

1. L.-H. Tranchau, Le Collège et le Lycée d' Orléans, 1762-1892, Orléans, H. Herluison, 1893 ; Bailly, Souvenirs d' enfance et de première jeunesse. Notes autobiographiques de l'helléniste orléanais conservées à la Bibliothèque municipale d'Orléans, Bulletin de la Société archéologique et historique de l'Orléanais, ${ }^{\circ}$ 65, 1984.

2. En 1902, Bailly habite rue Bannier, au $n^{\circ} 91$, tandis que Duvau demeure au $\mathrm{n}^{\circ} 97$.

3. Il y assiste aux cours du sanscritiste Abel Bergaigne, des latinistes Gaston Boissier, Émile Châtelain, Louis Havet, Roger Lallier, Othon Riemann, des hellénistes Olivier Rayet, Alfred Jacob, Henri Weil.

4. BnF, NAF 24439, f. 6.

5. Il touche 4000 francs et il n'a guère là l'espoir de dépasser 5000 francs. On lui propose 2000 francs. On comprendra qu'à la mort de Riemann, Édouard Tournier veuille lui faire obtenir la conférence de latin dont L. Havet (qui touche 3000 francs à cette époque) prendra finalement la charge (l'abbé Lejay ayant été débouté). Pour donner une idée, le traitement de S. Lévi s'élève à 12000 francs en 1896 par cumul des charges au Collège de France et à l'École. 
en réponse à celle que je lui avais adressée il y a quinze jours. Il me dit que faute d'avoir pu prévenir M. Meillet avant son départ pour l'Arménie, il se considère en quelque mesure comme moralement engagé à lui donner son appui, et ajoute, fort aimablement, que s'il avait été prévenu à temps, il n'aurait pas agi de cette façon.

Il faut donc conclure que pour être nommé à Paris, il faut y être déjà. Je ne pouvais poser ma candidature à une place qui n'était pas vacante : et celle de M. de Saussure ne l'est que depuis lundi dernier : je n'en ai même été avisé que ce matin par un mot de M. Châtelain. Quand j'ai appris que M. de Saussure était décidé à quitter Paris, il y a quinze jours, je lui ai écrit ; mais j'avais cru plus que déplacé que de faire allusion à son départ avant ce moment. Il me semble de plus que les arrangements pris dans ces conditions n'engagent personne que M. de Saussure, et en particulier, que le fait que M. Meillet a été suppléant un an déjà ne constituerait un titre sérieux qu'au cas où il y aurait eu à ce moment concurrence, ce qui n'a pas eu lieu et ne pouvait avoir lieu $[\ldots]^{6}$.

Aussi, et dans le même temps, Émile Châtelain argumente-t-il auprès de G. Paris :

[...] En outre je reçois sur l'École des échos que personne n'entend. Aux universités de Baltimore ou de Nebraska, les jeunes gens qui veulent faire de l'érudition connaissent tous les noms de notre affiche. Aucun séminaire de l'Allemagne ne possède notre admirable ensemble, tantôt c'est un historien, tantôt un philologue qui donne la célébrité à un de ces séminaires, mais des Allemands et Autrichiens sincères regardent notre École comme supérieure à tout ce qu'ils possèdent.

Dans ces conditions, j'ai trouvé les patrons de Meillet d'une rare modestie, voisine d'un certain mépris pour l'École, en proposant dès maintenant sa nomination. Et ce n'est pas une simple conjecture de ma part ; j'ai été humilié en 1889 quand beaucoup de jeunes gens, venus s'inscrire pour de Saussure, ont trouvé un enseignement tout différent, fort peut-être (mais aussi clair que celui de W. Meyer[-Lübke ?]). De Saussure accorde que M[eillet] n'a pas encore prouvé ce qu'il peut faire ; « la gutturale » qu'il a enseignée un an, qu'il présente en 38 pages pour être diplômé et qu'on n'a pas osé nous montrer encore, est, avec 7 pages d'arménien dans la Soc[iété] de linguistique, tout ce qu'il a produit ${ }^{7}$. À l'origine de l'École, on a nommé Maspero, Bergaigne, Guyard avec aussi peu de titres, je le veux bien, on a eu la main heureuse, mais leurs nominations ne brisaient la carrière de personne. En 1891, l'École est trop célèbre pour qu'une vocation soit un titre suffisant à prendre place sur notre affiche pour 40 ans. [...] La grande force de l'École consiste en ce que chacun n'enseigne que ce qu'il sait dans la perfection. J'admets que M[eillet] est ou sera le premier arménologue de l'Europe ; il suivra ses goûts en traitant des «langues du Caucase ». Ces conférences, «falsus utinam vates sim » [si seulement j'étais mauvais prophète !], ne se feront peut-être pas tous les ans, il y a des exemples pour ces enseignements spéciaux, ces « épaves » comme dit Boissier. Nous avons fait place au zend, à l'éthiopien, à l'hébreu rabbinique, à cause du mérite supérieur des titulaires, de leurs titres incontestables, mais on n'avait pas à craindre la critique de l'Europe et de 1'Amérique ${ }^{8}$.

Duvau, recruté en grammaire comparée à l'École, partagera la charge avec Antoine Meillet. Il affirme «s'inspirer de la méthode qu'avait suivie à différentes reprises dans son enseignement son éminent prédécesseur [...] il procèd[e] par voie analytique, et essai[e] de montrer comment, en partant de l'état historique du gothique [sic], on p[eut] distinguer les différentes lois qui depuis l'unité indoeuropéenne jusqu'à l'époque germanique, puis proprement gothique, avaient donné à la langue l'aspect qu'elle présente au IVe siècle $^{9} »$. Meillet, avec lequel il partage les heures, étudie lui les lois phonétiques du sanskrit. L'un et l'autre donnent deux conférences par semaine. En 1893, Duvau traite de la phonétique du gotique « en s'inspirant de la méthode qu'avait suivie, à différentes reprises, son éminent prédécesseur, M. Ferdinand de Saussure », et ainsi de suite. Directeur-adjoint à l'École des hautes études de 1891 à 1902, il remplace Bréal le deuxième semestre 1900-1901 au Collège de France et le deuxième semestre 1901-1902, et il le supplée en 1902-1903. Dans ces conférences, Duvau s'ouvre de plus en plus à l'étude de la

\footnotetext{
6. BnF, NAF 24439, f. 7-8.

7. Il s'agit de « De quelques difficultés de la théorie des gutturales indoeuropéennes » $(M S L P, 8,1894)$ et de « Notes arméniennes » $(M S L P, 8)$, 1892,15 p. Meillet soutiendra sa thèse en 1897. Comme Victor Henry et Louis Léger font partie du jury, ils ne peuvent rendre compte au public de ce travail dans la Revue critique. Meillet propose donc à Arthur Chuquet de confier le compte rendu de la thèse latine (De Indo-Euroaea radice *men- "mente agitare », 62 p.) à Grammont et celui de la thèse française Du génitif-accusatif en vieux-slave à P. Boyer (BnF, NAF 13694, f. 84).

8. BnF, NAF 24442, 27 juillet 1891, f. 132-135.

9. Annuaire de l'École des Hautes-Études, Paris, 1893-1895, p. 74.
} 


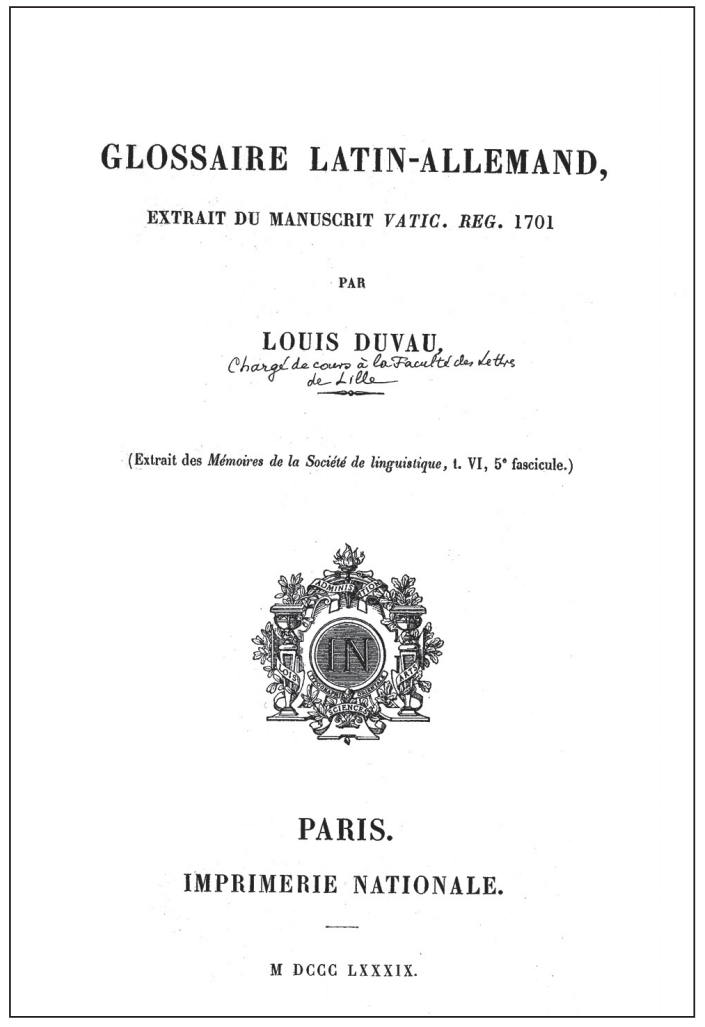

Fig. 1 : Page de titre du Glossaire latin-allemand extrait du manuscrit Vatic. Reg. 1701 (Mémoires de la Société de linguistique de Paris, 1889)

mythologie germanique et plus spécialement scandinave ; il ne s'occupe plus seulement de l'étude des langues mais il s'intéresse à la lecture des textes, encouragé dans cette démarche par Auguste Barth et par Bréal ${ }^{10}$. Mais Duvau meurt.

Collaborateur de diverses revues, directeur de la Revue de philologie de 1888 à 1897, secrétaire de rédaction de la Revue celtique de 1897 à 1901, administrateur de la Société de linguistique de Paris de 1892 à sa mort (il a 39 ans), il avait rassemblé une importante bibliothèque que bien après sa mort, en 1923, sa mère donne à la ville de Saumur (Duvau est enterré à Saumur) : 3026 numéros essentiellement voués à la littérature linguistique, riche d'ouvrages de philologie classique, de grammaire, de linguistique romane, nordique, orientale. Si le portrait de Louis Duvau a aujourd'hui disparu, il subsiste toutefois trois boîtes à archives, quelques travaux, des préparations de cours et de la correspondance ${ }^{11}$.

C'est de l'été 1888 que date la correspondance retrouvée entre Saussure et Duvau (Saussure a 31 ans, Duvau 24). Elle porte essentiellement sur des corrections que Saussure fait sur les épreuves que Duvau lui soumet d'un Glossaire latin-allemand extrait du manuscrit Vatic. Reg. 1701, écrit au XI $\mathrm{x}^{\mathrm{e}}$ siècle, qu'il vient de découvrir à Rome. L'étude sera publiée en 1889 dans les Mémoires de la Société de Linguistique de Paris (fig. 1), il y aura un tiré à part de quinze pages, et elle sera dédiée « à Ferdinand de Saussure. Hommage reconnaissant 12 ». Elle fait suite à la publication dans les Mélanges de l'École française de Rome du manuscrit latin $1701^{13}$, du fonds de la reine Christine au Vatican.

Saussure a été consulté pour sa compétence spéciale, parce que le glossaire présente des mots latins copiés à la suite, et que le copiste a ajouté, entre les lignes, d'une écriture plus fine, l'équivalent ou l'explication de ces mots. Or la plus grande partie de ces mots latins sont glosés par un mot allemand (et un petit nombre par un autre mot latin). «Un coup d'œil suffit, écrit Duvau dans la présentation de sa publication, pour reconnaître que c'est le haut-allemand, d'une époque notablement antérieure à celle où a été copié le Reginensis» qui

10. Ce dernier fait valoir l'avance prise par les pays voisins en ce domaine, si bien qu'en 1901, il souhaite transformer en sa faveur le cours de Léon Marillier, tout juste décédé, et qui était consacré à l'étude des religions des peuples non civilisés (Archives nationales F17 23300).

11. La Bibliothèque municipale de Saumur (Maine-et-Loire) a fait multigraphier le catalogue du fonds Louis Duvau dont le classement avait été effectué par Mlle Orieux, chargée de mission par la Direction des bibliothèques de France. Le don est évoqué dans le périodique de la Société des lettres, sciences et art du Saumurois, 1923, p. 15-18. Pour la biographie de Duvau, j’ai utilisé le Bulletin de la Société de linguistique de Paris, t. XIII, 1903-1905, p. LXV et 233 ; Émile Perrein, Bulletin de la Société des lettres, sciences et arts du Saumurois, 1923, fasc. II, p. 14-18 ; C. Port, Dictionnaire historique, géographique et biographique de Maine-et-Loire et de l'ancienne province d'Anjou, t. I, Angers, 1965, p. 94 et M. Prévost et Roman d'Amat, Dictionnaire de Biographie française, Paris, Letouzey et Ané, t. XII, 1948, p. 1008-1009.

12. T. VI, $5^{\mathrm{e}}$ fasc., Paris, imp. nat., 1889.

13. T. VIII, $5^{\mathrm{e}}$ fasc. 
constitue la langue des gloses germaniques. De février à août 1888, Saussure envoie quatre lettres, en tout quarante-sept pages.

La première lettre, datée du 24 février 1888, envoyée de Paris, compte quatre pages. Saussure atteste que Duvau a dû trouver un manuscrit inédit après qu'il a eu fait le tour des inventaires, celui de Steinmeyer-Sievers, dont les deux volumes alors parus sont en l'occurrence jugés inutiles (le troisième, assure Saussure, traitera de ces « répertoires, ou vocabulaires, et spécialement des vocabulaires qui sont disposés par classe d'objets » dont relève le présent manuscrit ${ }^{14}$ ), celui de Paul Piper (Die Sprache und Literature Deutschlands, 1880). Saussure a vérifié aussi qu'aucune glose romaine n'avait été publiée, ni dans la Zeitschrift für deutsches Alterthum und deutsche Litteratur 1880-1887, dirigée par Steinmeyer depuis des années, ni dans la bibliographie annuelle établie par Karl Bartsch dans la Germania de 1880 à 1884 au chapitre «Handschriftenkunde ». Saussure recommande cependant à Duvau de vérifier les années 85 , 86 et 87 , « momentanément sorties de la Bibliothèque de l'Université », et d'écrire éventuellement à Steinmeyer, « à moins que vous ne craigniez, écrit-il, de sa part une manœuvre diabolique qui consisterait à vous dire : nous connaissons depuis lgtmps vos gloses, elles sont sous presse en ce moment - et à dépêcher un jeune Teuton pour prendre copie du ms. 1701 ?? ». S'ensuivent quelques interrogations sur des formes qui, prétend Saussure, piquent sa curiosité, parce qu'elles sont rares ou énigmatiques.

La seconde lettre, du 10 août 1888, de huit pages, provient d'Yvoire-par-Sciez (Haute-Savoie). Ce sont des corrections après que Duvau eut déjà vérifié par deux fois sur le manuscrit ; certaines sont portées directement sur l'épreuve, d'autres sont par Saussure notées tout au long de cette lettre. Il s'agit d'établir au mieux les formes, de corriger les erreurs et d'apprécier les variantes qui auraient pu être introduites par le copiste. Des mentions marginales tracées par Duvau apprécient la nature de la correction : "Adm[is], Non, Rev[oir], Je corrige, B[ien], Bon, ms. ?, Voir... » Pour ne donner que deux exemples : "Comprenez-vous porro ? Je ne puis m'empêcher de supposer que c'est storio, l'allemand stûro étant l'esturgeon. Chose singulière on voit revenir col. 5, ligne 45-46 : suro-porro (et cette fois sans $t$ dans la forme allemande ?) »; « Non », est-il porté en marge ; «Il est bizarre de trouver zeiochr pour zeichor » : Duvau a là corrigé pour la publication.

La troisième, datée du 12 août, compte sept pages ; elle est commencée à Genève et achevée à Yvoire-parSciez. Elle est l'occasion de quelques «petits addenda », d'autant qu'étant venu à Genève, Saussure a pu consulter le Du Cange pour une forme qui fait problème (celle du mot latin leuga, répondant à slito, « traîneau »; Saussure ne trouve aucune solution si ce n'est de rapprocher qu' « à Genève les petits trâneaux sur lesquels les enfants s'amusent à se glisser sur la neige du haut d'une pente s'appellent des luges. Votre leuga est sûrement l'origine de ce mot local. Ce n'est pas la seule glose, ainsi que vs l'avez remarqué vs-même, qui soit intéressante au point de vue roman... »). Si les deux précédentes lettres n'étaient qu'amicales par courtoisie, en quelques mots de conclusion, celle-ci diffère. Deux pages sont plus chargées d'affects :

Je suis profondément ému, en vous écrivant, de la nouvelle de la mort tragique de $\mathrm{M}$. Bergaigne que je n'ai apprise qu'il y a quelques heures, et que vous aurez ressentie comme moi. Les journaux ne donnent pas encore beaucoup de détails, et je vois seulement que le malheureux M. Bergaigne a dû s'aventurer sans guide au milieu des rochers où il aura peut-être été surpris par la nuit. Il avait été pris depuis quelques années d'une passion de la montagne qui lui a été fatale [...] mais je ne puis m'empêcher de déplorer qu'il ait choisi, cette année, pour but d'excursion une région peu fréquentée des alpinistes, et par cela même plus dangereuse ; il aurait fait avec beaucoup moins de risque des ascensions beaucoup plus périlleuses dans les endroits où l'on ne part jamais sans guide, comme à Chamonix, ou à Zeermatt, dont il me parlait encore récemment ${ }^{15}$. C'est une perte aussi navrante que celle que nous faisions il y a quatre ans, presque jour pour jour, dans la personne de M. Guyard16, et toutes les deux également imprévues...

14. Il s'agit de Die Althochdeutschen Glossen gesammelt und bearbeitet von Elias Steinmeyer und Edward Sievers, Berlin, Weidmannsche Buchhandlung, 1879-1922, 5 vol. Les trois volumes auxquels il est fait allusion sont : I. Glossen zu biblischen Schriften, 1879 ; II. Glossen zu nichtbiblischen Schriften, bearbeitet von Elias Steinmeyer, 1882; III. Sachlich geordnete Glossare, bearbeitet von Elias Steinmeyer, 1895. 15. Rappelons que l'arrière-grand-père Horace-Bénédict de Saussure (1740-1799) est considéré comme le fondateur de l'alpinisme pour avoir précisément exploré cette région.

16. Stanislas Guyard (1846-1884), professeur d'arabe au Collège de France. 

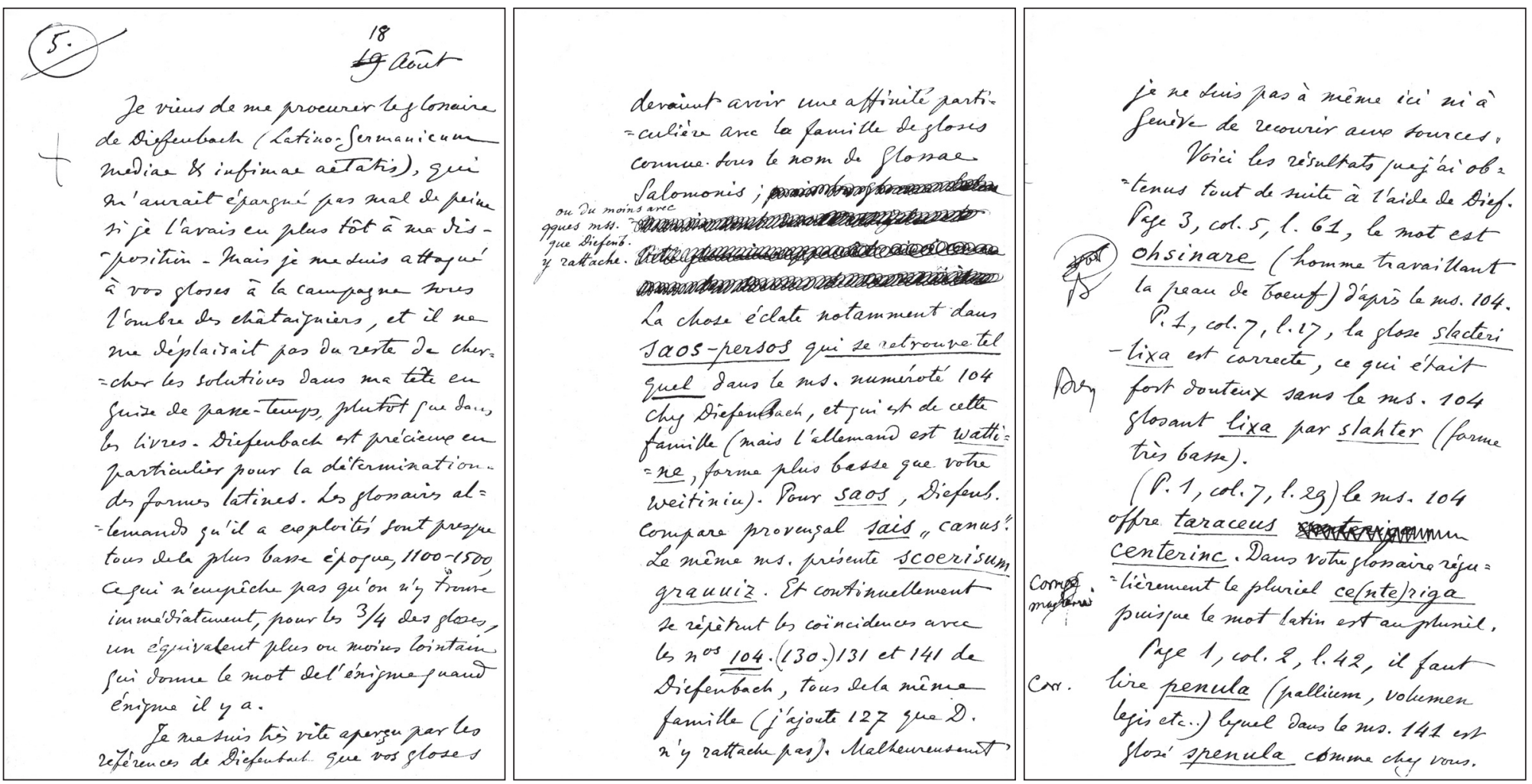

Fig. 2 : Lettre de Ferdinand de Saussure à Louis Duvau, 18 août 1888 (Bibliothèque municipale de Saumur)

On sait que la mort de Bergaigne à La Grave (HautesAlpes) affectera profondément le cénacle des comparatistes à l'École des hautes études, et notamment Georges Guieysse, l'élève préféré de Saussure17.

La dernière lettre, des 17-18 août et 20 août (d'Yvoire, voir fig. 2), est de loin la plus longue. Elle compte vingthuit pages. Ce sont la suite et la fin des notes de Saussure. Il y fait des rectifications, une suggestion et il fournit des « restitutions certaines », que Duvau annote toujours en marge, selon. Saussure vient aussi de se procurer le Glossaire de Lorenz Diefenbach (Latino-Germanicum mediae \& infimae aetatis, 1867) qui «m'aurait épargné pas mal de peine si je l'avais eu plus tôt à ma disposition. Mais je me suis attaqué à vos gloses à la campagne sous l'ombre des châtaigniers, et il ne me déplaisait pas du reste de chercher les solutions dans ma tête en guise de passetemps, plutôt que dans les livres. Diefenbach est précieux en particulier pour la détermination des formes latines. Les glossaires allemands qu'il a exploités sont presque tous de la plus basse époque, 1100-1500, ce qui n'empêche pas qu'on n'y trouve immédiatement, pour les 3/4 des gloses, un équivalent plus ou moins lointain qui donne le mot de l'énigme quand énigme il y a ». La suite de la lettre est occupée de restitutions.

Le feuillet daté du 20 août fait état du fait que Duvau et Saussure se sont ratés deux jours auparavant à Genève et Saussure affirme se réatteler sans délais à la tâche. Il revient par exemple sur porro ; "c'est un nom de poisson connu. Ma conjecture de storio est donc inutile ». Diefenbach lui est très utile pour revenir sur un certain nombre d'hypothèses émises, dont Duvau tentait de faire le tri. Enfin Saussure regrette qu'il soit désormais impossible à Duvau de rien vérifier sur le manuscrit. «Pour un certain nombre de corrections presque sûres il vaudrait toutefois la peine de mettre en note "lege ceci ou cela". Il serait aussi bien désirable que vous puissiez prendre connaissance du glossaire que Dief. désigne sous le $n^{\circ} 104$ et qui a certainement une parenté étroite avec le vôtre (lequel lui est bien antérieur)... »

Saussure conclut cordialement la lettre.

17. M. Décimo, «Une petite famille de travailleurs autour de Georges Guieysse : le monde de la linguistique parisienne », Cahiers Ferdinand de Saussure, n 52, Mélanges en hommage à Claudine Normand, 1999, p. 99-121. 
En apprenant la nomination de Duvau et de Meillet après lui, Saussure, qui écrit aimablement à Gaston Paris en décembre 1891, se réjouit :

[...] La nouvelle de la double nomination de M. Meillet et de M. Duvau reçue après mon départ de Paris, était de celles auxquelles je m'attendais le moins, comme sans doute tout le monde, et qui pouvaient le plus contenter le cœur d'un linguiste ami des deux candidats. Je ne doute pas, Monsieur et cher Maître, que cette solution n'ait été également conforme à votre désir, si vous ne l'avez même provoquée comme il est permis de le supposer. Je souhaite que personne n'ait lieu de se plaindre que l'atmosphère de l'École soit maintenant saturée de linguistique ; le mérite des deux titulaires garantit le contraire, mais c'est un grand honneur qu'on a fait à cette branche d'études ${ }^{18}$.

Saussure et Duvau resteront liés. Lors du Congrès des Orientalistes, Duvau résidera chez Saussure à MalagnyVersoix, près de Genève, comme l'atteste une lettre du 1'r septembre 1897 de Duvau à G. Paris. Duvau révise l'édition de Virgile établie par Eugène Benoist chez Hachette ; il est aussi l'auteur d'un traité de métrique en collaboration avec Louis Havet et de plusieurs articles, notamment sur la mythologie scandinave. Sa nécrologie dans l'Annuaire 1904 de l'ÉPHÉ est rédigée par Bréal et Émile Châtelain. Louis Finot ${ }^{19}$ écrit à Bréal de Phnom-Penh le 9 septembre [1903] qu'il a « appris avec regret la mort de Duvau ; c'était un esprit distingué qui n'a pas donné toute sa mesure. Avec lui disparaît un de vos héritiers et il doit vous en coûter d'autant plus d'abandonner votre chaire du Collège de France. Mais je comprends qu'après tant d'années si bien remplies vous éprouviez le besoin de vous reposer en contemplant la moisson que vous avez fait lever ${ }^{20} »$.

Deux lettres que G.-F. Möhl (qui va bientôt suppléer à Bréal au Collège de France et mourir à 38 ans) expédie de Prague, où il est alors maître de conférences en philologie romane et latin vulgaire à l'Université tchèque de Prague, apprennent à Bréal qu'il a lui-même beaucoup souffert d'un rude hiver et qu'il a subi une grave opération chirurgicale 21 . Il attend d'être employé à Marseille par une importante société d'huiles minérales pour ses connaissances en allemand. Il toucherait le même traitement qu' « un professeur impérial et royal ».

L'exemple de mon cher et infortuné Louis Duvau m'a enseigné la sagesse ; lui aussi, le pauvre garçon, a fait de la vie une expérience cruelle et la linguistique lui a été encore plus inclémente qu'à moi-même. Il y a trois ans lors de mon passage à Paris, je lui rendis visite dans son triste et misérable logement du quai de Béthune et, à le retrouver dans ce milieu lugubre, si changé, avec ses cheveux gris et son visage amaigri et souffrant, j'eus le pressentiment d'un malheur prochain. Je l'engageai à aller se reposer à la campagne ; mais il ne répondit que par un geste vague, un haussement découragé des épaules et qui, soulignant de précédentes allusions, quelques mots amers qui lui étaient échappés, disait toute la lassitude et les déceptions de la vie.

Je sortis de chez lui stupéfait et rêveur ; car à cette époque j'ignorais encore que, si la curiosité scientifique est le seul idéal vraiment digne de remplir la vie, il faut avant tout s'assurer les moyens de vivre et que la science ne les procure plus aujourd'hui qu'à un très petit nombre de ses élus ${ }^{22} \ldots$

18. M. Décimo, «Saussure à Paris », Genève, Cahiers Ferdinand de Saussure, ${ }^{\circ} 48,1994$, p. 79.

19. Cet élève de S. Lévi, L. Finot (1864-1935), diplômé de l'ÉPHÉ en 1894, chargé de la conférence de sanskrit en 1895, installe ce qui deviendra l'École française d'Extrême-Orient. En 1907, Finot obtient la chaire d'histoire et de philologie indochinoises au Collège de France. 20. Sorbonne, Ms 1967, f. 22-23.

21. Sorbonne, Ms 2289, f. 25. Ces lettres sont datées du 3 octobre 1902 et du 29 décembre 1903.

22. Sorbonne, Ms 1669, f. 43-46.

Maître de conférences à l'université d'Orléans, Régent du Collège de 'Pataphysique, chaire d'Amôriographie littéraire, ethnographique et architecturale, MARC DÉcimo est linguiste, sémioticien et historien d'art. Il a publié de nombreux articles sur la sémiologie du fantastique, sur l'histoire et l'épistémologie de la linguistique, et une vingtaine de livres, notamment sur Jean-Pierre Brisset (dont il a édité l'œuvre complète aux Presses du réel), sur Paul Tisseyre Ananké, sur Marie Le Masson Le Golft, sur Marcel Duchamp : La Bibliothèque de Marcel Duchamp, peut-être; Marcel Duchamp mis à nu. À propos du processus créatif; Le Duchamp facile; les mémoires de Lydie Sarazin-Levassor ; Marcel Duchamp et l'érotisme.

marc.decimo@gmail.com 